\title{
ELECTRONIC STRUCTURE SEEN BY POSITRONS IN EXTENDED AND REDUCED ZONE SCHEMES
}

\author{
G. Kontrym-Sznajd \\ W. Trzebiatowski Institute of Low Temperature and Structure Research \\ Polish Academy of Sciences \\ P.O. Box 937, 50-950 Wrocław 2, Poland \\ AND H. SORMANN \\ Institut für Kernphysik, Technische Universität Graz \\ Petersgasse 16, 8010 Graz, Austria
}

\begin{abstract}
The influence of the positron on the momentum distribution of annihilation quanta is investigated. Basing on general considerations, we show that a noninteracting positron, which generally reduces electronic densities, may enlarge some particular electronic umklapp components. Numerical tests were performed for alkalis, $\mathrm{Al}, \mathrm{Cu}$ and $\mathrm{Pd}$ by applying augmented plane wave band structure calculations. In the paper we discuss also the influence of this effect on the electron-positron densities after including the electron-positron correlation effects.
\end{abstract}

PACS numbers: 71.25.Pi, 71.60.+z, 78.70.Bj

\section{Introduction}

Due to the electron-positron interaction, the electron density at the positron site is enhanced. In momentum space it leads to a strongly momentum dependent enhancement factor of the electron momentum density. This problem was solved for the electron gas [1], but it is still ambiguous how it should be solved in the case of real metals.

It is known that the behaviour of umklapp densities (called also higher momentum components (HMC)) strongly depends on the theoretical approach used for description of the electron-positron correlation effects, e.g. the local density approach (LDA) [2] leads to either a de-enhancement or an over-enhancement [3] while the Bloch-modified ladder (BML) expansion approach always leads to a de-enhancement of the HMC densities [4]. Due to this reason the experimental investigation of the HMC enhancement could be utilized for a verification of these approaches. Of course, it requires a precise calculation of $\rho^{\mathrm{IPM}}(p)$, where IPM 
denotes the independent particle model. However, according to our theory [5], in some regions of $p$ the two-particle density $\rho^{\mathrm{IPM}}(p)$ can be evaluated with a high accuracy, largely independent from uncertainties arising from different approximations of the wave function $\psi_{+}(\boldsymbol{r})$. It allows to study the behaviour of the HMC without being afraid that possible disagreement between theory and experiment is connected with improper IPM calculations due to uncertainties arising from approximation of the positron wave function. It should be noticed that these "stable" densities correspond to densities of the highest value which can be really studied in the experiment, i.e. this important property of $\rho^{\mathrm{IPM}}(\boldsymbol{p})$ can be utilized for a verification of various approaches used for describing the electron-positron (e-p) interaction in real metals.

\section{Theory}

According to our results [5], in a periodic lattice potential the e-p momentum density (within IPM and in the $j$-th band) can be written as

$$
\rho_{j}^{\mathrm{IPM}}(k+G)=n(k j)\left|u_{k_{j}}\left(G_{k_{j}}\right)\right|^{2}\left|v\left(G-G_{k_{j}}\right)\right|^{2}\left[1+\alpha_{k_{j}}(G)\right]^{2},
$$

where

$$
\alpha_{k_{j}}(\boldsymbol{G})=\sum_{\boldsymbol{H} \neq \boldsymbol{G}_{\boldsymbol{k}_{j}}} \frac{v(\boldsymbol{G}-\boldsymbol{H}) u_{k_{j}}(\boldsymbol{H})}{v\left(\boldsymbol{G}-\boldsymbol{G}_{k_{j}}\right) u_{k_{j}}\left(\boldsymbol{G}_{k_{j}}\right)}
$$

and $u_{k j}$ and $v$ are the coefficients of the Fourier expansion of the electron and thermalized positron wave function, respectively. $G_{k j}$ is the reciprocal lattice vector $\boldsymbol{G}$ describing the leading coefficient with the property $\left|u_{\boldsymbol{k}_{j}}\left(\boldsymbol{G}_{\boldsymbol{k}_{j}}\right)\right|>\left|u_{\boldsymbol{k}_{j}}(\boldsymbol{H})\right|$. In order to make our formulae more clear, henceforth the index $k j$ in $\alpha, u$ and $x$ will be omitted.

In the case of $G=G_{k_{j}}$, corresponding to the leading term of the density

$$
\alpha\left(\boldsymbol{G}_{k_{j j}}\right)=x \max _{\left[\boldsymbol{H}_{\neq} \boldsymbol{G}_{k_{j}}\right]}\left|\frac{u(\boldsymbol{H})}{u\left(\boldsymbol{G}_{k_{j}}\right)}\right|,
$$

where $|x| \leq 1, \alpha\left(\boldsymbol{G}_{k_{j}}\right)$ is always considerably smaller than 1 .

For $\boldsymbol{G} \neq \boldsymbol{G}_{k_{j}}$, describing the umklapp components of $\rho(k+\boldsymbol{G})$,

$$
\alpha(\boldsymbol{G})=\frac{v(\mathbf{0}) u(\boldsymbol{G})}{u\left(\boldsymbol{G}_{\boldsymbol{k}_{j}}\right) v\left(\boldsymbol{G}-\boldsymbol{G}_{\boldsymbol{k}_{j}}\right)}\left[1+x u_{\max } / u(\boldsymbol{G})\right]
$$

with $u_{\max }$ defined as $\max \left[\boldsymbol{H}_{\neq} \boldsymbol{G}, \boldsymbol{G}_{\boldsymbol{k}_{j}}\right]^{|u(\boldsymbol{H})|}$ and $|x|$ lower than unity [5c, d]. For a few first umklapp densities (densities of the highest value) it holds $\left|x u_{\text {max }} / u(\boldsymbol{G})\right|<1$.

As we showed in the papers [5], such description of a two-particle density (Eq. (1)) and knowledge of general properties of $\alpha$ parameters allows to determine some general rules connected with features of $\rho(p)$. 
It is well known that the noninteracting positron reduces the electronic densities which is connected with Bunikowski-Schwarz inequality, i.e.

$$
\left\|\psi_{+} \phi_{k_{j}}\right\| \leq\left\|\psi_{+}\right\|\left\|\psi_{k_{j}}\right\|
$$

where $\|\psi\|$ denotes the norm of the function $\psi$. Therefore, if both electron and positron wave functions are normalized to the unity, the e-p momentum density satisfies the condition

$$
\sum_{G} \rho_{j}^{\mathrm{IPM}}(k+G) \leq 1
$$

As a result, $\rho^{\mathrm{IPM}}(p)$ after Lock-Crisp-West (LCW) folding (Ref. [6]) is not the same step function as in the case of the electron density, being additionally momentum-dependent (this effect was studied, on the example of $\mathrm{Nb}$, in the paper [7]).

In this paper we study the following effects:

1) If our theory, describing some general features of $\rho(p)$ [5], could explain also the results found by Kaiser et al. [7] as well as in the papers [8, 9].

2) If all components of $\rho_{j}^{\mathrm{IPM}}(k+G)$ are reduced, i.e. if

$$
\varepsilon_{j}^{\mathrm{IPM}}(k+\boldsymbol{G})=\rho_{j}^{\mathrm{IPM}}(k+\boldsymbol{G}) / \rho_{j}^{\mathrm{e}}(k+\boldsymbol{G})
$$

is less than unity or it can be also greater than unity leading to an intensification of the IIMC. Here the index e describes the electronic density and $\rho_{j}^{\mathrm{e}}(\boldsymbol{k}+\boldsymbol{G})=$ $\left|u_{k_{j}}(\boldsymbol{G})\right|^{2}$.

3) What kind of effects for $\rho(p)$ (both in $\boldsymbol{p}$ and $\boldsymbol{k}$ space) are expected after including to the IPM correlations effects.

The case $\boldsymbol{G}=\boldsymbol{G}_{\boldsymbol{k}_{j}}$, describing the density inside the Fermi surface (FS) in the extended zone scheme, and $\boldsymbol{G} \neq \boldsymbol{G}_{\boldsymbol{k}_{j}}$, providing HMC, are considered separately. In the next paragraphs the index $j$ in $\varepsilon, \rho, \xi$ and $\eta$ will be omitted, keeping in mind that all these considerations are done for a chosen band $j$.

$$
\text { 2.1. } \varepsilon^{\mathrm{IPM}}(k+G) \text { for } G=G_{k j}
$$

In this case, according to Eqs. (1) and (5),

$$
\varepsilon^{\mathrm{IPM}}\left(k+G_{k_{j}}\right)=|v(0)|^{2}\left|1+\alpha\left(G_{k_{j}}\right)\right|^{2} .
$$

Taking into account that $|v(0)|<1$ and $\left|\alpha\left(\boldsymbol{G}_{k_{j}}\right)\right|$ is a small number, $\varepsilon^{\mathrm{IPM}}\left(k+\boldsymbol{G}_{k_{j}}\right)$ can be greater than unity for such states $k j$ for which $\alpha\left(\boldsymbol{G}_{\boldsymbol{k}_{j}}\right)$ has a positive value as well as fulfils the inequality

$$
\alpha\left(\boldsymbol{G}_{\boldsymbol{k}_{j}}\right)>1 /|v(0)|-1 \text {. }
$$

According to our theory [5c, d], $\alpha\left(\boldsymbol{G}_{k_{j}}\right)$ is a negative number only for pure $d$ states. Therefore, on a base of general considerations, in this case we can expect that only for pure $d$ states the main component of the density will be reduced while for other states it could be intensified if the above condition for $\alpha$ is satisfied. However, because $|v(0)|$ is close to unity, usually positive $\alpha$ should satisfy this condition, particularly, for such states $k j$ for which the lattice effects are high (i.e. Fourier coefficients $u(\boldsymbol{H})$, belonging to umklapp components, are increasing). Particularly, $\varepsilon^{\mathrm{IPM}}\left(k 千 G_{k_{j}}\right)$ will be greater than 1 and relatively high for delocalized states 

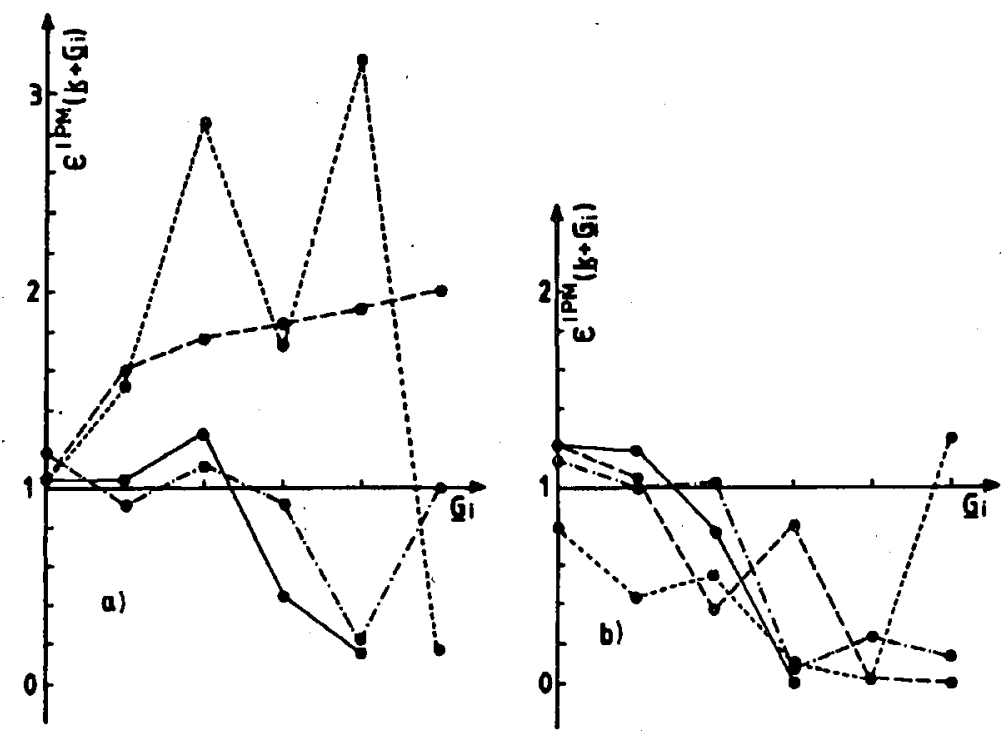

Fig. 1. $\varepsilon^{\mathrm{IPM}}(\boldsymbol{k}+\boldsymbol{G})$ for $\boldsymbol{G}=\boldsymbol{G}_{\boldsymbol{k}_{j}}$ (points on the vertical axis) and for a few first umklapp components ( $G=G_{1}, G_{2}, \ldots$, etc.) marked by points. These points were joined to guide the eyes. $\boldsymbol{G}_{\boldsymbol{i}}$ correspond to such reciprocal lattice vectors for which $\left|u\left(G_{i}\right)\right|>\left|u\left(G_{i-1}\right)\right|$. (a) In simple metals: $\mathrm{Li}, \mathrm{Na}, \mathrm{Cs}$ and $\mathrm{Al}$ in the first band $\left(\boldsymbol{G}_{\boldsymbol{k}_{j}}=\right.$ $(0,0,0))$ described by full, dashed, dashed-dotted and dotted lines, respectively. Here $k=(0.4,0.4,0.0)(\mathrm{Li}, \mathrm{Na}, \mathrm{Cs})$ and $k=(0.35,0.35,0.35)$ in $\mathrm{Al}$, where $k$ and $\boldsymbol{G}$ are given in units of $2 \pi / a$. (b) In Pd for $s$-, spd-, $d$-dominant and $d$-states shown by full, dashed, dashed-dotted and dotted lines, respectively. Here $G_{k_{j}}=(0,0,0)$ and $k=(0,0,0)$ (1st band, s-), $G_{k_{j}}=(0,0,0)$ and $k=(0.65,0,0)$ (6th band, spd-), $G_{k_{j}}=(0,0,0)$ and $k=(0.8,0,0)$ (1st band, $d$-domin.) and $G_{k_{j}}=(-2,0,0)$ and $k=(0.3,0,0)(5$ th band, $d-$ ).

in transition metals while in simple metals this effect will be increasing with $k$ approaching the Fermi momentum. It is confirmed by our numerical calculations (see Fig. 1). It is also obvious that the ratio

$$
\frac{\varepsilon^{\mathrm{IPM}}\left(\boldsymbol{k}+\boldsymbol{G}_{\boldsymbol{k}_{j}}\right)}{\varepsilon^{\mathrm{IPM}}\left(\boldsymbol{q}+\boldsymbol{G}_{\boldsymbol{q}_{j}}\right)}=\left|1+\frac{\alpha\left(\boldsymbol{G}_{\boldsymbol{k}_{j}}\right)-\alpha\left(\boldsymbol{G}_{\boldsymbol{q}_{j}}\right)}{1+\alpha\left(\boldsymbol{G}_{\boldsymbol{k}_{j}}\right)}\right|^{2}
$$

is close to unity in the case of simple metals, i.e. noninteracting positron does not change remarkably (in comparison with $\rho_{j}^{\mathrm{e}}\left(\boldsymbol{G}_{k_{j}}\right)$ ) the momentum dependence of $\rho_{j}^{\mathrm{IPM}}\left(\boldsymbol{G}_{\boldsymbol{k}_{j}}\right)$ (it was also discussed in Ref. [5a]).

$$
\text { 2.2. } \varepsilon^{\mathrm{IPM}}(k+G) \text { for } \boldsymbol{G} \neq \boldsymbol{G}_{k_{j}}
$$

In this case

$$
\varepsilon^{\mathrm{IPM}}(k+G)=|v(0)|^{2}|1+\xi(G)|^{2},
$$


where

$$
\xi(\boldsymbol{G})=\frac{u\left(\boldsymbol{G}_{k_{j}}\right) v\left(\boldsymbol{G}-\boldsymbol{G}_{\boldsymbol{k}_{j}}\right)}{u(\boldsymbol{G}) v(\mathbf{0})}+\frac{x u_{\max }}{u(\boldsymbol{G})} .
$$

Because $\xi$ cannot have a high negative value, i.e. $\xi$ cannot be lower than -2 , $\varepsilon^{\mathrm{IPM}}(\boldsymbol{k}+\boldsymbol{G})$ can be greater than unity only for such states $k j$ for which $\xi$ is a positive number and

$$
\xi>1 /|v(\mathbf{0})|-1 \text {. }
$$

Usually, for a few first HMC the second term in $\xi$ is negative (compare Eqs. (2) and (8)). Therefore in order to have $\xi>0$, the first term in $\xi$ must be both positive and greater that the second one. This can occur for some first umklapp components of $s$-like bands while for $d$ states, where mostly $\xi$ is a negative number, $\varepsilon^{\mathrm{IPM}}$ is lower than unity.

It is known that from angular correlation of annihilation radiation (ACAR) experiments we do not get absolute values of $\rho(p)$. Therefore, it is useful to define relative quantities, e.g.

$$
\eta^{\mathrm{IPM}}(\boldsymbol{G})=\frac{\varepsilon^{\mathrm{IPM}}(k+G)}{\varepsilon^{\mathrm{IPM}}\left(k+G_{k j}\right)}=\frac{1}{1+\alpha\left(G_{k j}\right)}|1+\xi(G)|^{2} .
$$

Of course, one could try to estimate from the experimental data the absolute value of the density by normalizing ACAR spectra to the total annihilation rates measured in lifetime experiments. Otherwise, we should remember that $\eta(k+G)$ could be either greater or lower than unity even if $\varepsilon(k+G)>1$.

\subsection{Generalization of the theory}

In the case of a real electron-positron (e-p) density, i.e. after including the correlation effects, the $\mathrm{e}-\mathrm{p}$ wave function can be written in the form

$$
\psi_{k j}^{\mathrm{e}-\mathrm{p}}(\boldsymbol{r}, \boldsymbol{r})=\tilde{\psi}_{\boldsymbol{k}_{j}}^{+}(\boldsymbol{r}) \psi^{\prime}{ }_{k j}(r),
$$

where, generally, the function $\tilde{\psi}[5 \mathrm{a}, \mathrm{b}]$ is defined as

$$
\tilde{\psi}_{k_{j}}^{+}(r)=f_{k_{j}}(r) \psi_{+}(r)
$$

This function is not normalized to unity and its norm strongly depends on the state $k j$ (in the case of the electron gas theory it is described by the enhancement factor $(\mathrm{EF}), \varepsilon_{\text {jell }}(p)$ ). The shape of the correlation factor $f_{k_{j}}(r)$ (equal to 1 in the IPM) could be very complicated, being dependent on other quantities (e.g. $\rho^{\mathrm{e}}(p)$, $\boldsymbol{G}$, etc.).

In the case of LDA [3], $f_{k j}^{\mathrm{LDA}}(r)$ has a similar shape as $\left|\psi_{+}(r)\right|$, i.e. it is a positive and monotonously increasing function of $r$. This is connected with the fact that $\rho(r)$ is decreasing function of $r$ and, as it is well known from lifetime measurements, the probability of the e-p annihilation is decreasing with increasing $\rho(r)$. Therefore, as previously, one can perform similar considerations for the functions $\tilde{\psi}_{\boldsymbol{k}_{j}}^{+}$and $\psi_{\boldsymbol{k}_{j}}$, replacing the coefficients $v$ by $\tilde{v}$ and studying $\varepsilon^{2 \gamma}=\rho / \rho^{\mathrm{e}}$ or for the function $f_{k_{j}}(r)$ and $\psi_{+} \psi_{k_{j}}$, studying $\varepsilon^{\text {corr }}=\rho / \rho^{\mathrm{IPM}}$. Of course, $\varepsilon^{\text {corr }}$ will 
be always greater than unity because the e-p interaction increases the probability of the e-p annihilation. This enhancement factor can be written as

$$
\varepsilon^{\operatorname{corr}}(k+G)=\left|\tilde{v}_{k j}(0)\right|^{2}|1+\tilde{\xi}(G)|^{2}
$$

(compare with Eq. (7)), where $\tilde{v}$ denotes the Fourier coefficients of the correlation factor $f_{k_{j}}(r)$ and the coefficients $u$ in $\tilde{\xi}$ are the Fourier coefficients of the e-p wave function $\psi_{+} \psi_{k_{j}}$ for the IPM. This equation clearly shows that the momentum dependence of this enhancement factor depends both on the state dependence of the correlation factor as well as on the lattice effects. Even if the state dependence of $f(r)$ is neglected, $\varepsilon^{\text {corr }}$ is momentum-dependent through the function $\tilde{\xi}$ (such effect has been pointed out in the paper [5a]). Therefore, $\eta_{\mathrm{LDA}}^{\text {corr }}$, similarly as $\eta^{\mathrm{IPM}}$, can be either greater or lower than unity, leading to either over-enhancement or de-enhancement effects of the HMC. Such numerical results were obtained for $\mathrm{Na}$ [3a] and $\mathrm{Mg}$ [3b] - the behaviour of the corresponding $\eta$ functions in $\mathrm{Na}$ is displayed in Fig. 2. Moreover, for a given band the function $\eta_{\mathrm{LDA}}^{\mathrm{corr}}$, in contrast to

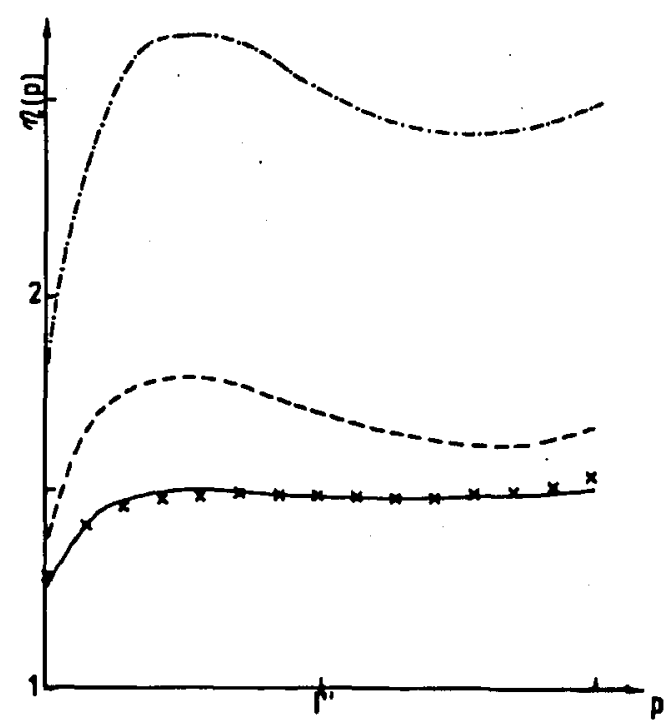

Fig. 2. Relative EF (function $\eta$ ) for $\mathrm{HMC}$ of valence electrons in $\mathrm{Na}$ along the [110] direction centred around the first reciprocal lattice vector $\Gamma^{\prime}$ for momenta $\Gamma^{\prime}-p_{\mathrm{F}}<$ $p<\Gamma^{\prime}+p_{\mathrm{F}}$ where $p_{\mathrm{F}}$ denotes the Fermi momentum. Full line and stars draw $\eta_{\mathrm{LDA}}^{\mathrm{cor}}$ when state dependence in the correlation factor $f_{\boldsymbol{k}_{j}}(\boldsymbol{r})$ was neglected and taken into account, respectively. Dashed and dashed-dotted lines show $\eta^{\text {IPM }}$ and $\eta^{2 \gamma}$.

$\varepsilon_{\mathrm{LDA}}^{\text {corr }}$, depends weakly on the state dependence of the correlation factor $f_{k_{j}}(r)$. It is connected with the fact that in the LDA the state dependence of the correlation factor is described by a dependence on the energy, which is the same for vectors $k$ and $k+G$.

In the case of transition metals one could expect that the local density dependence of the correlation factor $f(\boldsymbol{r})$ will cause (in contrast to BML approach) 
some additional effects also for the main components of densities if they are coming from differently localized bands. This effect can be read from Fig. 1 which illustrates that introducing an additional, position dependent function into calculations of $\rho(p)\left(\psi_{+}(r)\right.$ in the IPM or $f_{k j}(r)$ for a correlated system) generates an additional change in contributions of differently localized bands. Correlations effects, similarly as noninteracting positron, decrease a probability of the e-p annihilation together with localization of the band. Due to this reason, LDA and BML will lead to different result also for densities inside the central Fermi surface. Therefore, in this case it is reasonable to study $\varepsilon^{2 \gamma}$ (or $\varepsilon^{\text {corr }}$ ) for total densities both inside the central Fermi surface and outside of it.

\section{Summary}

In this paper we showed that noninteracting positron which reduces the $\mathrm{e}-\mathrm{p}$ densities folded into the 1st Brillouin zone ( $k$ space) can enlarge some particular components of this density (both main components and HMC) in the extended $p$ space. Values of $\varepsilon^{\mathrm{IPM}}\left(k+G_{k j}\right)$, which describe changes of the main components of $\rho^{\mathrm{e}}$, depend on the character of the $j$-th band being lower than unity only for localized states. The e-p local density correlation effects will lead to a similar result, having their reflection in the reduced $k$ space. As a result they will introduce an additional distortion of the LCW folded densities $[6,7]$ from the step function. Particularly, the density originating from localized bands will be more reduced. IIowever, the state dependence of the correlation factor will increase the contribution of these states $k j$ which are crossing the Fermi surface. This effect, in contrast to the previous one, increases a jump of $\rho(k)$ around the Fermi momentum which is helpful in drawing the Fermi surface map.

As concerns experimental studies of the correlation effects, up to now there is no answer what kind of physical effects is more important for a proper description of the e-p interaction in real metals. The interpretation of the experimental data in alkalis [4e] testified de-enhancement effect, in accordance with BML predictions. On the other hand, the interpretation of the reconstructed densities in $\mathrm{Mg}$ [3b] pointed out the over-enhancement of HMC (HMC of the third band along [110] direction), even stronger than it follows from the theoretical LDA calculations. Of course, all these investigations were connected with studies of very subtle effects because contributions of HMC in simple metals are very small. Due to this fact we rather recommend investigations of transition metals, studying the shape of the densities in the whole momentum space $p$. Moreover, we do not recommend to fit the EF to experimental densities (e.g. $\rho^{\exp }(p)=\sum_{j} \varepsilon_{j}(p) \rho^{\mathrm{IPM}}(p)$, where $\varepsilon_{j}(p)=$ $\sum_{m} c_{m}^{j} \mu^{m}$ and $\mu$ could be chosen as e.g. $\left.k, p, E_{j}(k)\right)$. Such a treatment needs a very precise fitting of all parameters, also $c_{0}^{j}$ which should change remarkably depending on the band and its proper estimation would have strong influence on the other parameters. Moreover, in the interpretation of these EF's one should remember that their state dependence $c_{m}^{j} \mu^{m} / c_{0}^{j}$ does not follow only from a state dependence of the correlation factor $\left(\tilde{v}_{k j}(0)\right.$ but also on the lattice effects described by the function $\tilde{\xi}$. 


\section{Acknowledgments}

The authors would like to express their thanks to Prof. R.N. West for very helpful discussion about the subject of this paper. This work is supported by the State Committee for Scientific Research (Republic of Poland) under grant No. 2 P302 16106.

\section{References}

[1] (a) H. Stachowiak, Phys. Rev. B 41, 12522 (1990); (b) E. Boroński, in: Positron Annihilation, Eds. Zs. Kajcsos, Cs. Szeles, Materials Science Forum, Vol. 105-110, Trans. Tech. Publ., Basel 1992; (c) A. Rubaszek, H. Stachowiak, in: Solid State Phenom. 28, 29, 9 (1993).

[2] S. Daniuk, G. Kontrym-Sznajd, J. Mayers, A. Rubaszek, H. Stachowiak, P.A. Walters, R.N. West, in: Positron Annihilation, Eds. P.C. Jain, R.M. Singru, K.P. Gopinathan, World Scientific, Singapore 1985, p. 43 and 279; J. Phys. F 17, 1365 (1987).

[3] (a) S. Daniuk, G. Kontrym-Sznajd, J. Majsnerowski, M. Sob, H. Stachowiak, J. Phys., Condens. Matter 1, 6321 (1989); (b) G. Kontrym-Sznajd, J. Majsnerowski, J. Phys., Condens. Matter 2, 9927 (1990).

[4] (a) K. Fujiwara, J. Phys. Soc. Japan 66, 1479 (1970); (b) K. Fujiwara, T. Hyodo, J. Ohyama, J. Phys. Soc. Japan 33, 1047 (1972); (c) B.B.J. Hede, J.P. Carbotte, J. Phys. Chem. Solids 33, 727 (1972); (d) H. Sormann, W. Puff, in Ref. [2], p. 161; (e) H. Sormann, Phys. Status Solidi B 142, K45 (1987); (f) H. Sormann, in: Positron Annihilation, Eds. L. Dorikens-Vanpraet, M. Dorikens, D. Segers, World Scientific, Singapore 1989, p. 37; (g) E. Boroński, T. Jarlborg, in Ref. [1], p. 607; (h) H. Sormann, D. Wallner, in Ref. [1], p. 845.

[5] (a) G. Kontrym-Sznajd, A. Rubaszek, Phys. Rev. B 47, 6950 (1993); (b) Phys. Rev. $B$ 47, 6960 (1993); (c) G. Kontrym-Sznajd, H. Sormann, submitted for publishing; (d) G. Kontrym-Sznajd, H. Sormann, Acta Phys. Pol. A 88, 185 (1995).

[6] D.G. Lock, V.H.C. Crisp, R.N. West, J. Phys. F, Met. Phys. 3, 561 (1973).

[7] J.H. Kaiser, R.N. West, N. Shiotani, J. Phys. F, Met. Phys. 16, 1307 (1986).

[8] L.P.L.M. Rabou, P.E. Mijnarends, Solid State Commun. 52, 933 (1984).

[9] A.K. Singh, R.M. Singru, J. Phys. F, Met. Phys. 14, 1751 (1984). 